\title{
Effectiveness of eHealth cardiac rehabilitation on health outcomes of coronary heart disease patients: a randomized controlled trial protocol
}

\author{
Jing Jing Su ${ }^{*}$ (D) and Doris Sau Fung Yu
}

\begin{abstract}
Background: Cardiac rehabilitation (CR) uptake and adherence remain sub-optimal despite the apparent health benefits of modifying healthy behavior and slowing disease progression. eHealth is the use of information and communication technology (ICT) for health. eHealth lifestyle interventions and disease management have emerged as modalities to enhance CR accessibility, enable an individualized progress page, and enrich real-time contact, video-based information, and technology monitored functionality. This study aims to develop a nurse-led eHealth cardiac rehabilitation (NeCR) intervention and investigate its effectiveness on coronary heart disease (CHD) patients' health outcomes.
\end{abstract}

Methods: This single-blinded two-arm parallel randomized controlled trial will randomize 146 patients from the inpatient cardiovascular units of a hospital in Wuhan, China to receive either the NeCR or the usual care. The NeCR intervention uses a hybrid approach consisting of a brief face-to-face preparatory phase and an empowerment phase delivered by health technology. The preparatory phase aims at identifying self-care needs, developing a goal-oriented patient centered action plan, incorporating a peer support network and orientation to the use of the e-platform. The empowerment phase includes use of the multi-media interactive NeCR for promoting symptom management, monitoring lifestyle changes and offering psychological support. A tele-care platform is also integrated to enhance health care dialogue with health professionals and peer groups. The control group will receive the usual care. An evaluation of lifestyle behavioral changes, self-efficacy, health-related quality of life, anxiety and depression, cardiovascular risk parameters, and unplanned health services use will be conducted at baseline, 6 weeks and 12 weeks post-intervention.

Discussion: This protocol proposes an individualized, comprehensive, and interactive NeCR delivered using a hybrid approach and guided by an empowerment model to optimize health outcomes of CHD patients. The intervention content and web-design is based on international health guidelines to improve credibility, comprehensibility and implementation. This study also proposes a new method of peer support in which the researcher shares participants' progress toward goal attainment with the peer group. Results of this research have the potential to increase accessibility and availability of $C R$, improve cardiac rehabilitation service development in China, and inform eHealth lifestyle interventions.

Trial registration: Chinese Clinical Trial Registry: ChiCTR1800020411; Date of registration: December 28, 2018. Keywords: eHealth, Cardiac rehabilitation, Coronary heart disease, Empowerment, Health behavior

\footnotetext{
* Correspondence: sujj@link.cuhk.edu.hk

Faculty of Medicine, The Nethersole School of Nursing, the Chinese

University of Hong Kong, Room 601, Esther Lee building, Shatin 999077,

Hong Kong
}

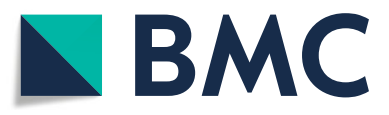

() The Author(s). 2019 Open Access This article is distributed under the terms of the Creative Commons Attribution 4.0 International License (http://creativecommons.org/licenses/by/4.0/), which permits unrestricted use, distribution, and reproduction in any medium, provided you give appropriate credit to the original author(s) and the source, provide a link to the Creative Commons license, and indicate if changes were made. The Creative Commons Public Domain Dedication waiver (http://creativecommons.org/publicdomain/zero/1.0/) applies to the data made available in this article, unless otherwise stated. 


\section{Background}

Coronary heart disease (CHD) is the accumulation of plaque in coronary arteries that limits blood perfusion of the heart [1]. It is related to cumulative exposure to risk factors as well as aging such that 40-year old men and women have a 49 and 32\% lifetime risk of developing $\mathrm{CHD}$, respectively $[2,3]$. An epidemiologic study indicated that patients with $\mathrm{CHD}$ had higher exposure to unhealthy lifestyle behaviors, and physiological and psychosocial risk factors [4]. Using effective behavioral change interventions to address these risk factors could prevent $75 \%$ of cardiac events and slow disease progression to heart failure or even death [5]. Current health statistics report 7.4 million deaths globally due to CHD and predict $\mathrm{CHD}$ will be the leading cause of disability by 2020 [6].

International guidelines strongly recommend cardiac rehabilitation (CR) programs to manage modifiable risk factors and improve health outcomes of CHD patients. The core components of CR incorporate physical exercise, patient education on lifestyle changes, disease management and psychosocial support [1, 7]. Indeed, CR is a structured program not only addressing direct CHD management but also other conditions including hypertension, dyslipidemia, diabetes and obesity. Substantial benefits associated with CR include reducing mortality by 20 to $47 \%$, reducing hospital re-admission by $18 \%$, improving physical activity, reducing cardiovascular risk factors and improving quality of life [1, 8-10].

Despite the apparent health benefits, $62 \%$ of countries do not offer CR, due to non-compatible health and social policy, inadequate infrastructure and manpower [11]. Rate of participation in and adherence to CR is also low and ranges from 14 to $35.5 \%$, due to inadequate access and time conflicts with other life activities [12, 13].

eHealth CR refers to the delivery of CR through information and communication technology (ICT), which has evolved as an alternative modality to improve availability and accessibility of CR. This mode includes a website, mobile applications, monitoring sensors, email, phone calls and short message services [14]. These make eHealth CR unique in being able to promote health behaviors in real-time so that participants can access information, upload self-monitored health data, receive automated feedback, and connect with peers or healthcare professionals [15]. eHealth CR also has the potential of being individually tailored to accommodate individual risk factors, goals and progresses using personal webpage for health behavior change [16]. It also enables experimental teaching that provides video-based health information to facilitate skill building. This modality is based on the statistic that $55.1 \%$ of the world's total population are Internet users [17]. Increasing participatory Internet use has been reported among older adults and become an important health information source and patient empowerment medium for health decision making and behaviors [18]. For this reason, there is increased advocacy to integrate eHealth platforms to deliver care components of CR to address the resource deficiency in traditional CR and provide more accessible and individualized health care to cardiac patients to improve health outcomes.

Existing evidence indicates high feasibility and acceptability of the e-platform to deliver CR $[14,15]$. A systematic review and meta-analysis was conducted to examine the effectiveness of eHealth CR on CHD patients [19]. Among the 14 studies reviewed [14, 20-32], meta-analysis indicated that eHealth CR led to significant improvement in physical activity, quality of life and re-hospitalization. There were inconclusive effects on diet, smoking, anxiety and depression, and other physiological risk parameters. This may be due to a lack of emphasis on promoting stress management, smoking cessation and symptom management among the study participants. As compared with studies which reported positive therapeutic effects on health behavioral outcomes, the studies which reported no positive effects were also less likely to incorporate the core strategies of patient empowerment to facilitate health behavioral changes. Such strategies are based on social cognitive theory and refer to individualized cardiovascular risk assessment, goal attainment process and enhanced self-directed tele-monitoring with professional feedback [19-24]. This review provides important insights on how to develop a more promising eHealth CR to enhance behavioral, psychological, physiological and clinical outcomes. All the identified studies in the review were conducted in developed countries including Australia [20], Belgium [21], Canada [22, 23], New Zealand [24, 25], Norway [26], Netherlands [27], Sweden [28], United Kingdom [29, 30], and the United States [14, 31, 32]. None were conducted in Asia/ China, where the cultural difference in health and illness behaviors and relationships with the health care system may limit the generalizability of the findings. China has 772 million Internet users with a penetration rate of 55.8 and $73 \%$ nationally and in urban areas, respectively [33]. The ability of older Chinese adults to use an eHealth intervention depends largely on a friendly webpage design, perceived usefulness and culture-compatible components $[34,35]$. Therefore, a randomized controlled trial is needed to investigate the effects of an individualized, empowerment-based, and culturally adjusted eHealth CR program to improve health outcomes for people with CHD.

\section{Social cognitive theory and patient empowerment}

The design of this eHealth CR is underpinned by social cognitive theory and patient empowerment model to enhance the translation of online input to actual health 
behavioral changes [36, 37]. This model uses a patientcentered collaborative approach that patients' perspectives, ability and resources will be assessed and integrated into formulating self-care goals and action plan. Interactive teaching and experimental learning will be used to enhance patients' knowledge and skills acquisition as well as transferring the knowledge and skills into behavior change. Self-regulation is also important as patients use proximal and distal goals to guide their behavior and monitor their health behavior and the circumstances under which it occurs.

\section{Aims}

This aims of the study are to develop a nurse-led eHealth cardiac rehabilitation $(\mathrm{NeCR})$ intervention and investigate its effects on lifestyle behavior, self-efficacy, health-related quality of life, anxiety and depression, cardiovascular risk parameters and unplanned health services use.

\section{Methods}

The study design is a single-blinded two-arm parallel randomized controlled trial comparing the effects of an eHealth CR with usual care on the behavioral and clinical outcomes of patients with CHD who are admitted for disease exacerbation.

\section{Study sample}

The study will be conducted at four cardiology units of Tongji Hospital, Wuhan, China where CHD patients are treated conservatively with percutaneous coronary intervention $(\mathrm{PCI})$ or medication and discharged under stable medication regimen. Eligibility requirements of participants are: adults aged 18 years or older; hospitalized for an initial diagnosis of CHD based on angiography or exacerbation of this condition in previously diagnosed cases; anticipated discharge to home; uses a computer and/or smartphone to access the Internet at home, reads and speaks Mandarin; minimum of primary education level; and no prescribed activity or exercise restriction. Exclusion criteria are: a diagnosis of acute psychotic disease/ life-limiting condition; absolute and relative contradictions to exercise testing and training and high risk for exercise prescription according to the American Association of Cardiovascular and Pulmonary Rehabilitation (AACVPR) guideline; and, having auditory, visual, fine motor, or ambulatory disorders.

Referring to a systematic review of studies which examined the effects of tele-cardiac rehabilitation on lifestyle behaviors, health outcomes and quality of life, the effects size on promoting healthy lifestyle is 0.42 to 1.29 so that a medium effect size of 0.5 (with $80 \%$ power and .05 significance) is conservatively adopted in this study [38]. The estimated sample size is 146, which takes into consideration an expected attrition rate of $12 \%$ based on prior studies with similar post-test time points of 6-12 weeks [31, 39]. Eligible participants will be identified by research nurses from four inpatient cardiac departments of the hospital by reviewing medical records. A second confirmation will be made with the on-site physicians. Eligible participants will then be invited to participate in this study and an information sheet will be provided. After obtaining informed consent, baseline data will be collected.

\section{Ethics approval}

Ethical approval for the study was received from the Joint Chinese University of Hong Kong - New Territories East Cluster Clinical Research Ethics Committee (2018.469). Approval was also obtained from the hospital. The study will be compliant with the Declaration of Helsinki. Protocol modifications will be communicated to the ethics committees.

\section{The nurse-led eHealth cardiac rehabilitation program}

The NeCR program is a health technology based CR program which aims to optimize positive behavioral changes and health outcomes of patients who are discharged following a CHD event. The intervention will be delivered by a cardiac nurse who has extensive experience in clinical teaching and patient education, using a hybrid approach in two phases: i) a brief face-to-face preparatory phase; and, ii) the CR program delivered by health technology using an empowerment approach. Since CR uses a multidisciplinary approach, cardiologists, cardiac nurse, physiotherapist and dietitian were involved in designing the content of the eHealth CR program. A nurse-led approach is used as the cardiac nurse plays a prominent role in supporting patient empowerment and health behavioral changes [40]. Nurses are effective in coordinating care with other health disciplines in rehabilitative services [41]. In implementing the eHealth CR program, the nurse researcher will coordinate the care and seek consultation with the involved disciplines if necessary to optimize the participants' outcomes. The intervention content is based on updated international guidelines and culturally appropriate national recommendations for CR [42-49].

\section{Face-to-face preparatory phase}

The preparatory phase will be conducted by the nurse researcher prior to the participant's discharge. It consists of two sessions, with the first session being an individualized health counseling session and the second as a group-based engagement session.

The aim of the individualized health counseling is to identify patients' self-care needs through a health assessment, develop client-centered goals and a corresponding 
action plan for lifestyle modification and disease management. The assessment focuses on self-care behaviors for managing CHD including exercise pattern, dietary habits, stress management and tobacco cessation as well as social aspects of disease management such as demographic background, role demand and social support to identify self-care needs. Patients' function capacity will be assessed by submaximal test using remote electrocardiography (Mindray Tel-200, China) monitored six-minute walk test, based on which the metabolic equivalent (MET) will be calculated by the nurse $[46,50]$. Accordingly, the nurse will provide brief health counseling based on the international guidelines recommendations for CR. The discrepancy between the patients' self-reported behaviors and the guideline recommendation will be highlighted, and the impact of this on health outcomes will be elaborated upon. By increasing the participants' awareness of their own self-care deficits, the nurse will support them in developing their goals for improving exercise patterns, dietary behaviors, and stress management.

The goal setting for exercise will indicate the frequency, duration, and intensity of exercise to gradually achieve at least $150 \mathrm{~min}$ of moderate weekly exercise. The nurse will also teach the participants about using Borg's ratings of perceived exertion to achieve the desired intensity with a perceived exertion of 12 to 14 ("somewhat hard" on a scale of 6 to 20) [51]. For dietary habits, a guideline based culturally appropriate 10 -item dietary habit checklist has been developed assessing food intake, cooking methods, eating pattern, and method of eating in restaurants. This checklist is adapted from the dietary checklist designed by the Department of Health in Taiwan to guide the dietary modification for cardiac patients [52]. The research team has modified the content so as to make it culturally relevant to the CHD patients in China. The revised content has been validated by two Chinese dietitians. Participants will be supported in setting successive goals to eventually attain over $90 \%$ adherence to this checklist. As for stress management, participants will be supported to set goals of managing stress below a level of 3 as assessed by $0-9$ scored scale [53]. The nurse will collaborate with the patients to work out the action plan with a variety of exercise, dietary and relaxation skills options. Participants who smoke will also be supported to set a cessation goal and provided with tips such as nicotine replacement therapy, avoid smoking for social influences and managing stress [54]. A written participant-centered goal-driven action plan and information handout for self-management will be provided to reinforce knowledge retention. The individualized goals and action plan will be uploaded to the participants' password protected personal webpages on the eHealth CR platform.
The group-based engagement session will take place in the hospital meeting room with $4 \sim 6$ participants per group. The aims are: i) orientate participants to the operation of the web- and tele- platform, and ii) form a cohesive peer support group to optimize lifestyle behavioral changes. The nurse will share anonymously the goals of the participants and discuss the importance of goal attainment in optimizing health outcome. The nurse will give an orientation to the web-platform by demonstrating each key feature and distribute the eplatform user manual. In order to ensure the success of the subsequent self-monitoring on physical exercise, a pedometer with written instructions, will be provided. Practice and return demonstration is needed to ensure skill mastery. Finally, the nurse will invite participants to enter a WeChat telecare platform consisting of $12 \sim 16$ participant members.

\section{CR program delivery by health technology using an empowerment approach}

The 12-week CR program is the core component of the intervention and aims at using two core methods including an interactive and motivational web platform, and a tele-care platform to optimize CR participation and behavioral modification.

The interactive and motivational web-based platform consists of three key platforms: a) self- monitoring with motivational feedback platform, b) an interactive and experiential learning platform, and c) health dialogue forum. The design of this platform followed the guideline of the Health Literacy Online to improve the comprehensiveness and utility of the content [55]. The content was revised to be at the readability level of sixth to ninth grade examined by Microsoft ${ }^{\circ}$ Word $^{\circ}$ Office Package (Microsoft Corp, Redmond, WA) to optimize comprehension. The web platform can be automatically adjusted to computer and smartphone interfaces to improve flexibility and access.

\section{Self- monitoring with motivational feedback platform}

The self-monitoring page will document the goals and action plans developed for improving exercise level, dietary habits, stress management and/ or tobacco cessation during the preparatory phase. Participants are recommended to input self-monitoring data on a daily/weekly basis to indicate the level of goal attainment. For exercise, participants will upload exercise times, the intensity level as light/moderate/ vigorous and the duration of engagement in 1 week. In addition, the guidelines for $\mathrm{CR}$ also recommend a higher level of walking to exceed 7000 steps [56], and each participant will be encouraged to upload their daily step count measured by their pedometer. Participants will be encouraged to complete the guideline based dietary habit checklist and their score on the 0 to 9 stress scale to indicate their 
dietary habit and stress level. A traffic light system will be used to give motivational feedback based on the level of goal attainment, with red indicating attention required $(<50 \%$ of goal attainment), yellow representing moderate achievement ( $>50-90 \%$ of goal attainment) and green indicating full attainment (90-100\% of goal attainment). An automated message which corresponds to the level of goal attainment and shares the trend in participants' recordings will be presented. Patients will be asked to report on tobacco use and encouraged to update their smoking status (if applicable) with green indicating they have quit while red represents smoking. Based on the pre-determined coding, an overall score on goal attainment will be generated along with the traffic light system to reflect progress on each behavioral change.

\section{Interactive and experiential learning platform}

The interactive and experiential learning platform provides all intervention group participants access to comprehensive health information regarding CHD. The content was developed according to the updated international guidelines and culturally appropriate national recommendations for CR by the American Heart Association, American Psychological Association, American Diabetes Association, The American Cancer Society, Chinese Nutrition Society, and the National Center for Cardiovascular Disease [42-49]. The content covers the pathophysiology and manifestation of CHD, physical activity, diet management, smoking cessation, stress management, risk factors management (hypertension, cholesterol, and diabetes), symptom management and postPCI management. The health information has been adapted to fit in the Chinese context, and the content of this platform has been validated by a cardiologist, dietitian and cardiac nurses. To motivate participants, each component is presented sequentially: i) introducing the role and underlying mechanism of each care component in disease management, ii) the required lifestyle changes, iii) recommended actions, and iv) self-monitoring to track progress and resolutions to barriers. Various learning opportunities will be provided using scenario-based case sharing to illustrate behavioral change barriers, challenges and resolution alternatives; videos for skills demonstrations and role modeling; and pictures, slogans and comics to present these sessions in an engaging manner. The health dialogue forum aims at providing a method for posting CR research news and answering questions from participants.

\section{Tele-care platform}

In the tele-care platform, the nurse will retrieve selfmonitoring data uploaded by participants and anonymously share goal attainment of group members as well as discuss their experiences and address any concerns.
Reminders for uploading data will be sent to participants weekly. This platform permits participants to contact the nurse about issues or adverse changes in health. The nurse will contact each individual at weeks 2 and 4 postdischarge to assess fitness, explore difficulties, encourage progress and offer solutions. This may lead to revision of the action plan or goal adjustment. Participants will be asked to maintain password and account security, and to avoid discussing of intervention content with other patients in the hospital to avoid contamination.

\section{Usual care}

Participants in the both groups will receive the usual health care information related to maintaining a healthy lifestyle (exercise, diet and smoking cessation), instructions on medication during hospitalization, and one follow-up call. The research nurse will instruct control group participants on use of the pedometer.

\section{Randomization and data collection procedure}

Eligible patients will be invited to participate in this study and an information sheet will be provided. After obtaining informed consent, baseline data will be collected. Participants will be randomly assigned to either the intervention or control group by block randomization. Three different block sizes of 4, 6 and 8 will be used. The random number for group assignment will be generated by random allocation software. The group assignment will be written and placed in an opaque sealed envelope and given to participants by the research assistant after baseline data collection $\left(\mathrm{T}_{0}\right)$. Outcome evaluation will take place at the 6-week intervention $\left(\mathrm{T}_{1}\right)$ and upon completion of the 12-week intervention $\left(\mathrm{T}_{2}\right)$ by trained research assistants who are blinded as to the participants' intervention allocation (Fig. 1). Participants will not be blinded as to their group allocation. The following parameters for outcome assessment will be used to include health behavior, emotional outcomes, health-related quality of life, cardiac physiological risk parameters and unplanned health services use. Participants' travel expenses for data collection will be reimbursed.

\section{Primary outcomes}

The primary outcomes focus on health behavioral change including physical activity, health promoting lifestyle habits, and smoking behaviors. Physical activity is defined as movement of the body that uses energy. Objectively, physical activity level will be measured by the pedometer (Mi Band, China) record of step count for three weekdays [57]. A pedometer correlates strongly with different accelerometers (median $r=0.86$ ) as well as the time in observed activity (median $r=0.82$ ) [58]. The International Physical Activity Questionnaire (IPAQ) will be used to comprehensively assess the self-reported 


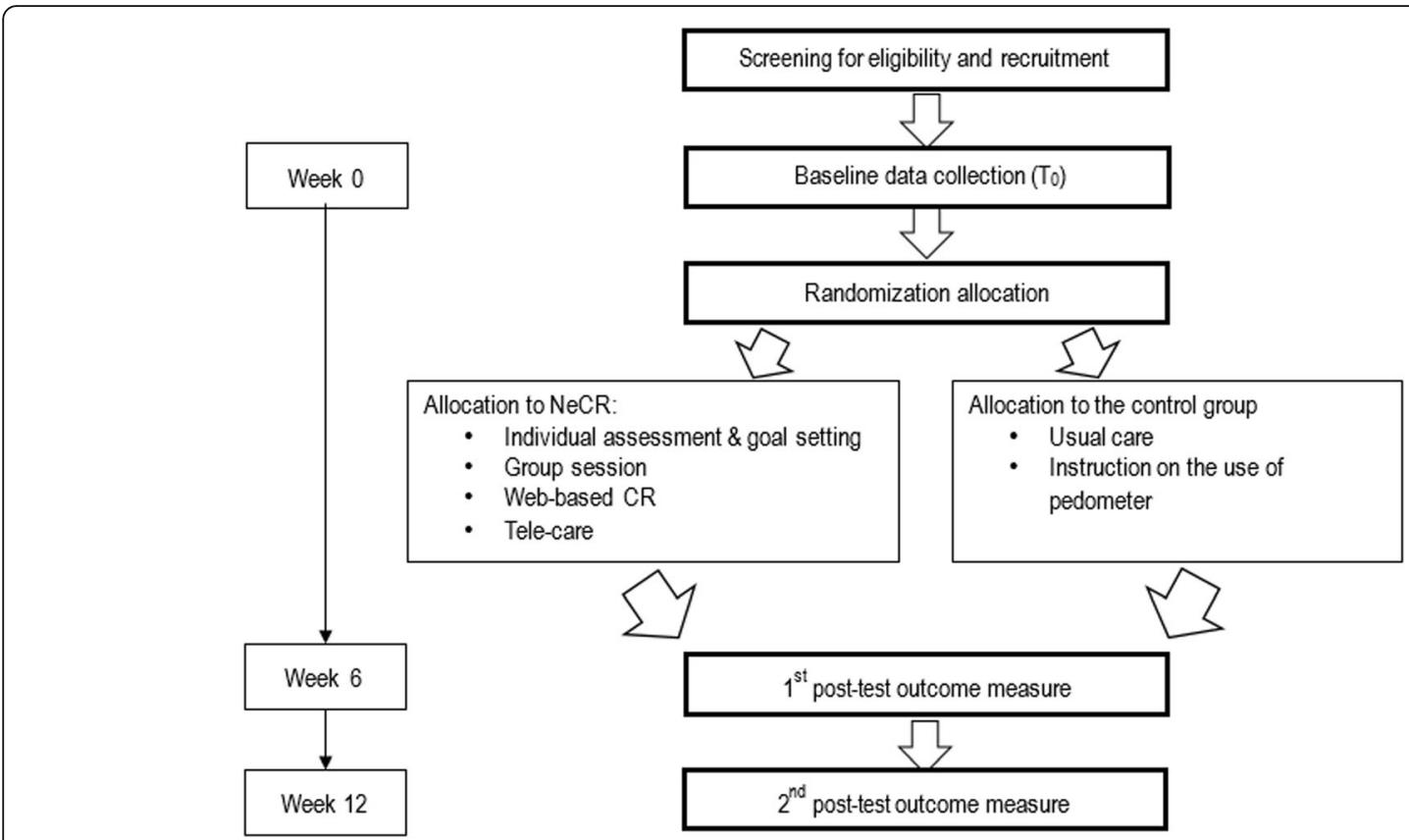

Fig. 1 Flow diagram of the study protocol

time spent in walking, light/moderate/rigorous exercise and sitting across various domains using 27 items. The IPAQ has test-retest reliability with intra-class correlation coefficients of 0.74 to 0.97 for each sub-domain for Chinese adults [59]. Total physical activity measured by the IPAQ was moderately correlated with pedometer measured exercise levels $(r=0.46)$ [60].

Health promoting behaviors will be measured by the Health Promoting Lifestyle Profile II (HPLP- II) [61]. The HPLP- II measures six aspects of health promotion behaviors including nutrition, interpersonal support, stress management, exercise, health responsibility and self-actualization. The HPLP - II has good internal consistency with 0.92 for the entire scale and $0.69-0.84$. The HPLP uses a four-point scale with 1-4 representing always to never with a total score ranging from 40 to 160 and a higher score indicating better health behavior. The HPLP-II has been translated and validated into Chinese with Cronbach's $\alpha$ coefficient of 0.63 to 0.81 [62].

Smoking status will be asked to evaluate the abstinence rate using two questions: (1) what is your current smoking status? (current smoker, ex-smoker or nonsmoker) (2) How many cigarettes do you smoke per day? [63].

\section{Secondary outcomes}

Cardiac self-efficacy refers to an individual's confidence in managing cardiovascular disease. It will be measured using the Cardiac Self-efficacy Scale (CSES), originally developed by Sullivan [64]. The 13 item questionnaire consists of two subscales to measure one's self-efficacy in maintaining function and to control symptoms. The CSES uses a 5-point Likert scale with a higher score indicate higher self-efficacy. The original version of the CSES has good internal consistency with Cronbach's alpha of 0.90 and 0.87 for the subscales. The CSES has been translated and validated among Chinese cardiac patients with internal consistency of 0.926 . The construct validity of the C-CSES was established with significantly moderate correlations General Self-efficacy Scale $(\mathrm{r}=$ $0.47, p<0.001$ ) [65].

Health-related quality of life will be measured by the MacNew Heart Disease health related quality of life questionnaire (HRQoL), which evaluates how an individual's emotional, physical and social well-being are affected by CHD and its treatment. It has 27 items scored from 1 (low HRQoL) to 7 (high HRQoL) with internal consistency and intraclass correlation coefficients $\geq 0.73$ [66]. It has been translated and validated among Chinese CHD populations with intraclass correlation coefficient ranging from 0.88-0.93 [67].

Psychological status of participants will be measured by the Depression Anxiety Stress Scale 21 (DASS-21) consisting of 21 items in three domains: depression, anxiety and stress [68]. Respondents are required to indicate the presence of the symptom(s) over the past week on a 4-point Likert scale scoring from 0 to 3 with higher scores indicate more severe symptoms. This instrument has well-established psychometric properties in reliably measuring depression, anxiety and stress (Cronbach's alpha of $0.91,0.84$ and 0.90 respectively). The DASS-21 correlated strongly with hospital anxiety and depression 
scale both for anxiety $(\mathrm{r}=0.88)$ and depression $(\mathrm{r}=0.93)$ [69]. The DASS-21 has been translated and validated among Chinese with Cronbach's alpha $\geq 0.80$ [70, 71].

The cardiac physiological risk parameters include body mass index (BMI), blood pressure and waist circumference. Body weight and height will be examined using the same equipment (Xiheng, RGZ-120-RT, China) with participants in light clothing, shoes removed, upright posture and looking straight ahead. Blood pressure will be measured in a sitting position after participants have rested for $10 \mathrm{~min}$ (Omron HEM-7124, Japan). Waist circumference will be measured using a flexible tape measure at umbilicus level.

The unplanned health services use is defined as unplanned cardiac-related hospital readmissions, emergency department visits and re-vascularization. Because China does not have an electronic medical records system, the participants will be reminded to call the outcome assessor whenever an unplanned health services event occurs. To reduce recall bias, the outcome assessor will assess participants' unplanned medical services use by monthly call asking about the detailed date, unit, diagnosis and related information. Survival analysis of time-to-event data will be conducted to describe the length of time from entering the program to any readmission.

\section{Demographic and clinical data}

Comprehensive demographic and clinical date will be collected at baseline to permit examination of factors that may influence the NeCR outcomes of participants. Demographic data (age, sex, education, marital status, employment status, living conditions and Internet use) will be collected from participants while clinical data will be retrieved from the medical record to include diagnosis, clinical presentation, documented hypertension, dyslipidemia, diabetes, any treatments being received and single/multiple vessel disease.

\section{Data management}

Participants will be given a code to ensure anonymity. According to Chinese University of Hong Kong Policy on Research, Intellectual Property and Knowledge Transfer, data will be stored in a locked computer accessible only to the principal investigator and researcher [72]. Data entry of all questionnaires will be validated by a second person.

\section{Data analysis}

The latest version of SPSS will be used to analyze data with a 5\% significance level (two-sided). Treatment evaluation will be performed based on the principle of intention to treat. A generalized estimating equations (GEE) model will be used to compare the differential between the two groups related to each of the outcomes across the time-points, with adjustment for potential confounding variables in order to obtain a more precise estimation of intervention effect. The potential confounding variables will be selected on the basis of clinical judgment and statistical incomparability at baseline. For the smoking behavior as nominal data, the Chi-square test will be used to compare the number of patients who quit smoking between the groups. In addition, the Friedman test will be used to compare the changes in percentage of smoking cessation among the groups over time [73]. Survival analysis, the analysis of time-to-event data will be conducted to describe the length of time from entering the program to an unplanned health services utilization.

\section{Process evaluation}

Experiences and perceptions of participants in the NeCR will be assessed through individual in-depth interview of a sample of 15-20 intervention group participants. Sampling will occur until theoretical saturation, which is expected to be reached by selection of $20 \%$ of the intervention participants $[74,75]$. To maximize the sample variation, participants who have different responses to the NeCR in terms of the changes on their scores on the primary outcomes $(0-35$ th percentile, $>35$ th percentile and $>70 \%$ percentile of the score) will be recruited. The subject selection will also attempt to optimize the relevant clinical and demographic characteristics such as age, gender, education and treatment received. Interviews will be conducted by a researcher with extensive experiences in qualitative interviewing and independent of outcome assessment. The interview will focus on individual NeCR experiences, perceived effectiveness, likes and dislikes and further suggestions. Thematic content analysis will be used including an iterative process reading, coding, categorizing and identifying themes [76].

Engagement with the $\mathrm{NeCR}$ will be assessed using the website $\log$ file for numbers of $\log$ in and data upload. Numbers of communication with the nurse for consultation and events or issues sharing among peers will be recorded.

\section{Potential risks management}

All personal electronic data will be stored in a passwordprotected account to ensure confidentiality. Participants have been informed that they will incur no additional costs for care received during the intervention and it will not replace care provided by their physician. Participation in the study will be discontinued if a participant's health status changes and no longer meets inclusion criteria and they will be referred to appropriate care services. To minimize any possible adverse event due to exercise, patients will be advised to start exercise at their metabolic equivalent level based on a six-minute walk 
test and increase gradually in frequency, duration, and intensity. Participants will be encouraged to access symptom management information and contact the nurse for guidance when needed. An emergency card with participant's name, diagnosis, contact information of the family/friend and researcher will be provided in case of an adverse event due to disease deterioration or exercise.

\section{Discussion}

This study will use a hybrid approach guided by an empowerment model to investigate the effect of eHealth CR which integrates behavior change strategies on not only exercise but also on diet, stress and smoking. The intervention content and web-design are based on international guidelines and national culturally appropriate recommendations to improve credibility, comprehensibility and implementation for this population. Individual assessment and a collaborative approach to goal setting and action planning helps participants understand the purpose of using the web- and tele-platform. This could also encourage participants' engagement in self-care decision making with their health care professional and proactive use of the e-platform, which are resources for self-management. Considering the continuous care needs of a patient discharged to their home setting after a cardiac event, individual consultation and guidance from a nurse and encouragement from peer interaction and support will be initiated through multi-communication channels as social motivators for behavioral goal attainment. This study uses a new method of peer support in which the researcher shares participants' progress toward goal attainment with the peer group and encourages participants to share their experiences and concerns to reinforce behavioral changes.

\section{Limitations of the study}

There are several possible limitations to this protocol. Although participants will be recruited from a regional hospital which serves a population with diverse demographic and socio-economic characteristics, the generalizability of the study findings will still be limited since the study involves only one province in China and excludes those residents who are illiterate. Another limitation is that self-report measures are used to monitor the level of participation in the eHealth CR and the changes in behavioral, psychological and health-related status. The findings may subject to social desirability and recall bias. Also, even though an attention placebo in the form of patient education is used in this clinical trial, none of the initiatives can ensure that an equal amount of attention is paid to both groups. This may result in a threat to internal validity.

\section{Conclusion}

This study reports the development and evaluation of a novel eHealth CR program for the Chinese CHD population. By using a hybrid approach to integrate minimal personal contact with intensive and multimodal eHealth initiatives, the eHealth CR program addresses the limited accessibility and acceptability of the conventional CR program without scarifying its merit in providing encounters with healthcare professionals. With the detailed information about the program content and implementation plan as well as the evaluation in the process and outcome perspectives, this paper provides insights to enable the model replication or application in the management of other chronic diseases.

\section{Abbreviation \\ AACVPR: American association of cardiovascular and pulmonary rehabilitation; BMI: Body mass index; CHD: Coronary heart disease; CR: Cardiac rehabilitation; CSES: Cardiac self-efficacy scale; DASS- \\ 21: Depression anxiety stress scale 21; GEE: Generalized estimating equations; HPLP- II: Health promoting lifestyle profile II; HRQOL: Health related quality of life questionnaire; IPAQ: International physical activity questionnaire; MET: Metabolic equivalent; NeCR: Nurse-led eHealth cardiac rehabilitation; $\mathrm{PCl}$ : Percutaneous coronary intervention}

\section{Acknowledgements}

None.

\section{Trial status}

Currently open for recruitment.

\section{Authors' contributions}

JJS and DSFY conceived and designed the study; JJS and DSFY designed the web prototype, sampling plan, data collection and analysis plan; JJS and DSFY drafted and finalized the article. All authors have reviewed and approved the final version of the manuscript being submitted. The article is the authors' original work has not been previously published and is not under consideration for publication elsewhere.

\section{Funding}

Individual-funded.

\section{Availability of data and materials}

Datasets from this study will be available from the corresponding author upon reasonable request.

Ethics approval and consent to participate

This study was approved by the Joint Chinese University of Long Kong-New Territories East Cluster Clinical Research Ethics Committee and Tongji Hospital. The study is compliant with Declaration of Helsinki. Ethics considerations are based on the principals of beneficence, respect for human dignity, confidentiality, and right to withdraw from the study at any time. All patients provided written, informed consent before the procedure and gave consent for personal information to be used in this study.

\section{Consent for publication}

Not applicable.

Competing interests

The authors declare that they have no competing interests. 
Received: 20 May 2019 Accepted: 15 November 2019 Published online: 29 November 2019

\section{References}

1. American Heart Association. What is Cardiac Rehabilitation? Apr 23,2015. 2016. http://www.heart.org/HEARTORG/Conditions/More/CardiacRehab/ What-is-Cardiac-Rehabilitation_UCM_307049_Article.jsp\#.Wp31Y6huaUk. Accessed 12 Jan 2018.

2. Benjamin EJ, Blaha MJ, Chiuve SE, Cushman M, Das SR, RD, et al. Heart disease and stroke statistics - 2017 update: A report from the American Heart Association. 2017.

3. Lloyd-Jones D, Adams RJ, Brown TM, Carnethon M, Dai S, De Simone G, et al. Heart disease and stroke statistics - 2010 update: a report from the American Heart Association. Circulation. 2010;121:e46-e215. https://doi.org/ 10.1161/CIRCULATIONAHA.109.192667.

4. Navar AM, Peterson ED, Wojdyla D, Sanchez RJ, Sniderman AD, D'agostino $\mathrm{RB}$, et al. Temporal changes in the association between modifiable risk factors and coronary heart disease incidence. JAMA. 2016:316:2041-3.

5. Wang W, Lau Y, Chow A, Thompson DR, He H-G. Health-related quality of life and social support among Chinese patients with coronary heart disease in mainland China. Eur J Cardiovasc Nurs. 2014;13:48-54. https://doi.org/10. 1177/1474515113476995.

6. World Health Organization. Media Centre: Cardiovascular disease: WHO; 2017. http://www.who.int/mediacentre/factsheets/fs317/en/. Accessed 10 Feb 2018

7. Eckel RH, Jakicic JM, Ard JD, De Jesus JM, Houston Miller N, Hubbard VS, et al. 2013 AHA/ACC guideline on lifestyle management to reduce cardiovascular risk: a report of the American college of cardiology/American heart association task force on practice guidelines. J Am Coll Cardiol. 2014; 63:25 PART B:2960-84.

8. Lawler PR, Filion KB, Eisenberg MJ. Efficacy of exercise-based cardiac rehabilitation post-myocardial infarction: A systematic review and metaanalysis of randomized controlled trials. Am Heart J. 2011;162:571-584.e2. https://doi.org/10.1016/j.ahj.2011.07.017.

9. Buckingham S, Taylor R, K J, A Z, SG D, A C, et al. Home-based versus centre-based cardiac rehabilitation abridged Cochrane systematic review and meta analysis Cochrane database Syst Rev 2016:8:CD007130.

10. Heran BS, Chen JM, Ebrahim S, Moxham T, Oldridge N, Rees K, et al. Exercise-based cardiac rehabilitation for coronary heart disease. Cochrane Database Syst Rev. 2011. https://doi.org/10.1002/14651858.CD001800.pub2.

11. Turk-Adawi K, Sarrafzadegan N, Grace SL. Global availability of cardiac rehabilitation. Nat Rev Cardiol. 2014;11:586-96. https://doi.org/10.1038/ nrcardio.2014.98

12. Ruano-Ravina A, Pena-Gil C, Abu-Assi E, Raposeiras S, Van't Hof A, Meindersma $\mathrm{E}$, et al. Participation and adherence to cardiac rehabilitation programs. A systematic review. Int J Cardiol. 2016;223:436-43. https://doi. org/10.1016/j.ijcard.2016.08.120.

13. Fang J, Ayala C, Luncheon C, Ritchey M, Loustalot F. Use of Outpatient Cardiac Rehabilitation Among Heart Attack Survivors - 20 States and the District of Columbia, 2013 and Four States, 2015. MMWR Morb Mortal Wkly Rep. 2017;66:869-73. https://doi.org/10.15585/mmwr.mm6633a1.

14. Widmer RJ, Collins NM, Collins CS, West CP, Lerman LO, Lerman A, et al. Digital Health Interventions for the Prevention of Cardiovascular Disease: A Systematic Review and Meta-Analysis. Mayo Clin Proc. 2015;90:469-480. https://doi.org/10.1016/j.mayocp.2014.12.026

15. Beatty AL, Fukuoka Y, Whooley MA. Using mobile technology for cardiac rehabilitation: a review and framework for development and evaluation. J Am Hear Assoc. 2013:2:e000568, https://doi.org/10.1161/JAHA.113.000568.

16. Yardley L, Choudhury T, Patrick K, Michie S. Current issues and future directions for research into digital behavior change interventions. Am J Prev Med. 2016;51:814-5. https://doi.org/10.1016/j.amepre.2016.07.019.

17. Internet World Stats. World Internet usage and population statistics. 2018. https://www.internetworldstats.com/stats.htm. Accessed 30 Oct 2018.

18. Medlock S, Eslami S, Askari M, Arts DL, Sent D, De Rooij SE, et al. Health information-seeking behavior of seniors who use the internet: a survey. J Med Internet Res. 2015;17:e10.

19. Su JJ, Yu DSF, Paguio JT. Effect of eHealth cardiac rehabilitation on outcomes of coronary heart disease patients: a systematic review and metaanalysis. In: Shaping the Future of Nursing: Pathway to serve the Public and Nursing Profession, Yonsei International Nursing Conference; 2018.
20. Varnfield M, Karunanithi M, Lee C-K, Honeyman E, Arnold D, Ding H, et al. Smartphone-based home care model improved use of cardiac rehabilitation in postmyocardial infarction patients: results from a randomised controlled trial. Heart. 2014;100:1770-9. https://doi.org/10.1136/heartjnl-2014-305783.

21. Frederix I, Hansen D, Coninx K, Vandervoort P, Vandijck D, Hens N, et al. Medium-term effectiveness of a comprehensive internet-based and patientspecific telerehabilitation program with text messaging support for cardiac patients: randomized controlled trial. J Med Internet Res. 2015;17:1-10.

22. Lear SA, Singer J, Banner-Lukaris D, Horvat D, Park JE, Bates J, et al. Randomized trial of a virtual cardiac rehabilitation program delivered at a distance via the internet. Circ Cardiovasc Qual Outcomes. 2014;7:952-9.

23. Reid RD, Morrin LI, Beaton LJ, Papadakis S, Kocourek J, McDonnell L, et al. Randomized trial of an internet-based computer-tailored expert system for physical activity in patients with heart disease. Eur J Prev Cardiol. 2012;19: 1357-64. https://doi.org/10.1177/1741826711422988.

24. Dale LP, Whittaker R, Jiang Y, Stewart R, Rolleston A, Maddison R. Text message and internet support for coronary heart disease self-management: results from the text4heart randomized controlled trial. J Med Internet Res. 2015:17(10):e237. https://doi.org/10.2196/jmir.4944.

25. Maddison R, Pfaeffli L, Whittaker R, Stewart R, Kerr A, Jiang Y, et al. A mobile phone intervention increases physical activity in people with cardiovascular disease: results from the HEART randomized controlled trial. Eur J Prev Cardiol. 2015;22:701-9.

26. Antypas K, Wangberg SC. An internet- and mobile-based tailored intervention to enhance maintenance of physical activity after cardiac rehabilitation: short-term results of a randomized controlled trial. J Med Internet Res. 2014;16:1-18.

27. Vernooij JWP, HAH K, van der Graaf Y, Wierdsma J, HMH G, MMC H, et al. Internet based vascular risk factor management for patients with clinically manifest vascular disease: randomised controlled trial. BMJ. 2012;344:e3750.

28. Johnston N, Bodegard J, Jerstrom S, Akesson J, Brorsson H, Alfredsson J, et al. Effects of interactive patient smartphone support app on drug adherence and lifestyle changes in myocardial infarction patients: a randomized study. Am Heart J. 2016;178:85-94.

29. Devi R, Powell J, Singh S. A web-based program improves physical activity outcomes in a primary care angina population: randomized controlled trial. J Med Internet Res. 2014;16:e186. https://doi.org/10.2196/jmir.3340.

30. Lindsay S, Smith S, Bellaby P, Baker R. The health impact of an online heart disease support group: a comparison of moderated versus unmoderated support. Health Educ Res. 2009;24:646-54.

31. Zutz A, Ignaszewski A, Bates J, Lear SA. Utilization of the internet to deliver cardiac rehabilitation at a distance: a pilot study. Telemed e-Health. 2007;13: 323-30. https://doi.org/10.1089/tmj.2006.0051.

32. Southard BH, Southard DR, Nuckolls J. Clinical trial of an internet-based case management system for secondary prevention of heart disease. J Cardpulm Rehabil. 2003;23:341-8 http://search.ebscohost.com/login.aspx?direct= true $\& \mathrm{db}=\mathrm{ccm} \& A N=106709383 \&$ site $=$ ehost-live\&scope $=$ site

33. CNNIC. 39th Statistical Report on Internet Development in China. CNNIC Statistiscal Reports. 2016. https://cnnic.com.cn/IDR/ReportDownloads/2015 07/P020150720486421654597.pdf. Accessed 12 Feb 2018.

34. Huang M, Hansen D, Xie B. Older adults' online health information seeking behavior. Proc 2012 iConference. 2012::338-45. doi:https://doi.org/10.1145/ 2132176.2132220

35. Wong CKM, Yeung DY, Ho HCY, Tse K-P, Lam C-Y. Chinese older adults' internet use for health information. J Appl Gerontol. 2014;33:316-35. https://doi.org/10.1177/0733464812463430.

36. Bandura A. Health promotion by social cognitive means. Heal Educ Behav. 2004;31:143-64

37. Zimmerman MA. Psychological empowerment : issues and illustrations. Am J Community Psychol. 1995;23:581-99.

38. Rawstorn JC, Gant N, Direito A, Beckmann C, Maddison R. Telehealth exercise-based cardiac rehabilitation: a systematic review and meta-analysis. Heart. 2016;102:1183-92.

39. Widmer RJ, Allison TG, Lennon R, Lopez-Jimenez F, Lerman LO, Lerman A. Digital health intervention during cardiac rehabilitation: a randomized controlled trial. Am Heart J. 2017:188:65-72.

40. Jiang $X$, Sit JW, Wong TK. A nurse-led cardiac rehabilitation programme improves health behaviours and cardiac physiological risk parameters: evidence from Chengdu. China J Clin Nurs. 2007;16:1886-97 http:// easyaccess.lib.cuhk.edu.hk/login?url=http://ovidsp.ovid.com/ovidweb.cgi?T= $J S \& C S C=Y \& N E W S=N \& P A G E=$ fulltext $\& D=$ med5\&AN $=17880478$. 
41. Fridlund $\mathrm{B}$. The role of the nurse in cardiac rehabilitation programmes. Eur J Cardiovasc Nurs. 2002;1:15-8.

42. American Heart Association. 3 Tips to Manage Stress. 2017. http://www. heart.org/en/healthy-living/healthy-lifestyle/stress-management/3-tips-tomanage-stress. Accessed 10 Sept 2018.

43. American Psycological Association. Psychology Help Center. 2018. http://www.apa.org/helpcenter/index.aspx. Accessed 10 Sept 2018.

44. Hu D-Y, Li N, Tong Q-G. Hypertension and hyperlipidemia_diet and exercise. Beijing: China Light Industry Press; 2017.

45. American Diabetes Association. Cardiovascular disease and risk management: standards of medical care in diabetes 2018. Diabetes Care. 2018;41:S86-104.

46. American Association of Cardiovascular and Pulmonary Rehabilitation. Guidelines for cardiac rehabilitation and secondary prevention programs. 15th ed. Champaign: Human Kinetics; 2013.

47. The American Cancer Society. Guide to Quitting Smoking: what do I need to know about quitting? 2014. p. 48.

48. Chinese Nutrition Society. Chinese Dietary Guidlines 2016. Beijing: People's Medical Publishing House; 2016.

49. National Center for Cardiovascular Diseases. Report on cardiovascular disease in China 2016. 2017; http://www.nccd.org.cn/Sites/Uploaded/File/2 017/8/中国心血管病报告2016中文(72).pdf. Accessed 10 Feb 2018.

50. Bittner $\mathrm{V}$. Role of the 6-minute walk test in cardiac rehabilitation. In: Kraus WE, Keteyian SJ, editors. Cardiac rehabilitation. Totowa: Humana Press; 2007. p. 131-9. https://doi.org/10.1007/978-1-59745-452-0_12.

51. Borg G. Borg's perceived exertion and pain scales. Champaign: Human Kinetics; 1998

52. Department of Health. Diet assessment for cardiac patients. 2018. https:// cloud.health.gov.tw/TPH_PHM_Cloud/App_Prog/Assessment.aspx?id=2. Accessed 3 Sept 2018

53. Young QR, Nguyen M, Roth S, Broadberry A, MacKay MH. Single-item measures for depression and anxiety: validation of the screening tool for psychological distress in an inpatient cardiology setting. Eur J Cardiovasc Nurs. 2015;14:544-51.

54. Yang T, Fisher KJ, Li F, Danaher BG. Attitudes to smoking cessation and triggers to relapse among Chinese male smokers. BMC Public Health. 2006;6:65.

55. Office of Disease Prevention and Health Promotion. Health Literacy Online. 2016. https://health.gov/healthliteracyonline/.

56. American College of Sports Medicine. ACSM'S Guidelines for Exercise testing and Prescription. Philadelphia: Lippincott Williams \& Wilkins; 2013.

57. El-Amrawy F, Nounou MI. Are currently available wearable devices for activity tracking and heart rate monitoring accurate, precise, and medically beneficial? Healthc Inform Res. 2015;21:315-20. https://doi.org/10.4258/hir. 2015.21.4.315

58. Tudor-Locke C, Williams JE, Reis JP, Pluto D. Utility of pedometers for assessing physical activity. Sport Med. 2002;32:795-808. https://doi.org/10. 2165/00007256-200232120-00004

59. MacFarlane D, Chan A, Cerin E. Examining the validity and reliability of the Chinese version of the international physical activity questionnaire, long form (IPAQ-LC). Public Health Nutr. 2011;14:443-50.

60. Vandelanotte C, De Bourdeaudhuij I, Philippaerts R, Sjöström M, Sallis J. Reliability and validity of a computerized and Dutch version of the international physical activity questionnaire (IPAQ). J Phys Act Health. 2005; 2:63-75.

61. Walker SN, Sechrist KR, Pender NJ. The health-promoting lifestyle profile: development and psychometric characteristics. Nurs Res. 1987;36:76-81.

62. Cao WJ, Chen CS, Hua Y, Li YM, Xu YY, Hua QZ. Factor analysis of a healthpromoting lifestyle profile (HPLP): application to older adults in mainland China. Arch Gerontol Geriatr. 2012;55:632-8. https://doi.org/10.1016/j. archger.2012.07.003.

63. Global Adult Tobacco Survey Collaborative Group. Tobacco questions for surverys: a subset of key questions from the global adult tobacco survey (GATS). 2nd ed. Altlanta: Center for Disease Control and Prevention; 2011. https://www.who.int/tobacco/surveillance/en_tfi_tgs.pdf

64. Sullivan MD, LaCroix AZ, Russo J, Katon WJ. Self-efficacy and self-reported functional status in coronary heart disease. Psychosom Med. 1998:60:473-8. https://doi.org/10.1097/00006842-199807000-00014.

65. Zhang X, Zhan Y, Liu J, Chai S, Xu L, Lei M, et al. Chinese translation and psychometric testing of the cardiac self-efficacy scale in patients with coronary heart disease in mainland China. Health Qual Life Outcomes. 2018; 16:1-8.
66. Hofer S, Lim L, Guyatt G, Oldridge N. The MacNew heart disease healthrelated quality of life instrument: a summary. Heal Qual Life Outcomes. 2004;2:3. https://doi.org/10.1186/1477-7525-2-3.

67. Thompson DR, Oldridge NB, Yu DSF, Yu CM. Translation and validation of two Chinese health-related quality of life instruments in patients with coronary heart disease. Hong Kong Med J. 2009;15(Suppl 2):8-11 http://www.ncbi.nlm.nih.gov/pubmed/19258626.

68. Lovibond PF, Lovibond SH. The structure of negative emotional states: comparison of the depression anxiety stress scales (DASS) with the Beck depression and anxiety inventories. Behav Res Ther. 1995;33:335-43.

69. Sukantarat KT, Williamson RCN, Brett SJ. Psychological assessment of ICU survivors: a comparison between the hospital anxiety and depression scale and the depression, Anxiety and Stress scale. Anaesthesia. 2007;62:239-43. https://doi.org/10.1111/j.1365-2044.2006.04948.x.

70. Zuo K, Chang A. A Chinese Translations of the DASS (simplified characters). 2008. http://www2.psy.unsw.edu.au/groups/dass/Chinese/Chinese simplified. htm. Accessed 11 Sept 2018.

71. Chan RCK, Xu T, Huang J, Wang Y, Zhao Q, Shum DHK, et al. Extending the utility of the depression anxiety stress scale by examining its psychometric properties in Chinese settings. Psychiatry Res. 2012;200:879-83. https://doi.org/10.1016/j.psychres.2012.06.041.

72. The Chinese University of Hong Kong. Policy on Research, Intellectual Property and Knowledge Transfer. 2017. http://www.orkts.cuhk.edu.hk/ images/Research_Funding/The_Policy_Paper_1b.pdf. Accessed 24 Apr 2019.

73. Munro BH. Statistical methods for health care research. Philadelphia: Lippincott Williams \& Wilkins; 2005

74. Kvale S, Brinkmann S. Interviews: Learning the craft of qualitative research interviewing; 2009. https://doi.org/10.1017/CBO9781107415324.004.

75. Risom SS, Lind J, McCabe P, Berg S. Patient perspectives of participating in the cardiac CopenHeartRFA rehabilitation program for patients treated with ablation for atrial fibrillation. J Multidiscip Healthc. 2018;11:167-74. https://doi.org/10.2147/JMDH.S152823.

76. Holloway, Immy and Galvin K. Qualitative research in nursing and healthcare edition 4th. Oxford: Wiley-Blackwell; 2016.

\section{Publisher's Note}

Springer Nature remains neutral with regard to jurisdictional claims in published maps and institutional affiliations.

Ready to submit your research? Choose BMC and benefit from

- fast, convenient online submission

- thorough peer review by experienced researchers in your field

- rapid publication on acceptance

- support for research data, including large and complex data types

- gold Open Access which fosters wider collaboration and increased citations

- maximum visibility for your research: over $100 \mathrm{M}$ website views per year

At $\mathrm{BMC}$, research is always in progress.

Learn more biomedcentral.com/submissions 